\title{
Ultracold bosonic atoms in two-colour superlattices
}

\author{
Robert Roth and Keith Burnett \\ Clarendon Laboratory, University of Oxford, Parks Road, Oxford OX1 3PU, UK
}

Received 21 September 2002, in final form 20 November 2002

Published 2 April 2003

Online at stacks.iop.org/JOptB/5/S50

\begin{abstract}
The influence of disorder on ultracold atomic Bose gases in optical lattices is discussed in the framework of the one-dimensional Bose-Hubbard model. It is shown that simple periodic modulations of the well depths generate a rich phase diagram consisting of superfluid, Mott insulator, Bose glass (BG) and spatially localized phases. The detailed evolution of mean occupation numbers and number fluctuations as function of modulation amplitude and interaction strength is discussed. Finally, the signatures of the different phases, especially of the BG phase, in matter-wave interference experiments are investigated.
\end{abstract}

Keywords: Bose-Einstein condensation, optical lattices, Bose-Hubbard model, Mott insulator phase

\section{Introduction}

Quantum phase transitions of interacting bosonic many-body systems in disordered lattice potentials have been a topic of intense theoretical investigations in the past years. A rich variety of possible zero-temperature phases has been predicted such as the Mott insulator (MI) phase, an Anderson localized phase, and a Bose glass (BG) phase [1-5]. Recently, impressive experiments on the transition from a superfluid (SF) to a MI [6] demonstrated that ultracold bosonic atoms in optical lattices offer unique possibilities to explore the phase diagram of these systems. The degree of experimental control is remarkable: the geometry of the lattice potential can be designed and specified precisely, even spatial modulations of the well depths can be introduced in a controlled manner [7, 8]. The strength of the two-body interaction can be chosen by means of Feshbach resonances. Ultimately, the structure of the ground state can be examined in detail. This makes them a promising candidate to study the competition between disorder and interaction experimentally in an unprecedented way.

In this contribution we discuss the ground state phase diagram of an interacting bosonic many-body system in an optical superlattice, i.e. a lattice composed of irregular unit cells. In particular, we investigate a sinusoidal modulation of the well depths as it can easily be produced by a superposition of two standing-wave lattices of different wavelengths, a socalled two-colour lattice. We study the dependence of the exact ground state on the interaction strength and the amplitude of the modulation within the Bose-Hubbard model.

\section{Bose-Hubbard model}

Consider a gas of $N$ bosonic atoms in a one-dimensional lattice potential at zero temperature. For a sufficiently strong lattice it is convenient to describe the state of the system in a basis of localized 'tight-binding' wavefunctions, i.e. the Wannier functions that result from a band structure calculation. We assume that only the localized ground state of each lattice well contributes and excited vibrational states can be neglected. For a system with $I$ lattice sites the many-body state can thus be represented in terms of number states $\left|n_{1}, \ldots, n_{I}\right\rangle$ with occupation numbers $n_{i}$ for the individual sites $i=1, \ldots, I$. A complete basis of the model space is formed by the set of number states $\left|n_{1}^{(\alpha)}, \ldots, n_{I}^{(\alpha)}\right\rangle(\alpha=1, \ldots, D)$ with all compositions of the occupation numbers. The dimension $D=(N+I-1) ! /[N !(I-1) !]$ of the number basis grows dramatically; for $I=8$ lattice sites and $N=8$ particles the dimension is 6435, for $I=N=10$ it is already 92378 . Within the model space any state can be expanded in this number basis

$$
|\psi\rangle=\sum_{\alpha=1}^{D} C_{\alpha}\left|n_{1}^{(\alpha)}, \ldots, n_{I}^{(\alpha)}\right\rangle
$$

with expansion coefficients $C_{\alpha}$. We introduce creation operators $\boldsymbol{a}_{i}$ and annihilation operators $\boldsymbol{a}_{i}^{\dagger}$, which create and annihilate respectively a particle in the lowest vibrational state at site $i$, and the corresponding occupation number operators $\boldsymbol{n}_{i}=\boldsymbol{a}_{i}^{\dagger} \boldsymbol{a}_{i}$ 
The Hamiltonian of the interacting many-body system in second quantization is the so-called Bose-Hubbard Hamiltonian [1-4]

$$
\boldsymbol{H}=\sum_{i=1}^{I}\left[-J\left(\boldsymbol{a}_{i+1}^{\dagger} \boldsymbol{a}_{i}+\boldsymbol{a}_{i}^{\dagger} \boldsymbol{a}_{i+1}\right)+\epsilon_{i} \boldsymbol{n}_{i}+\frac{V}{2} \boldsymbol{n}_{i}\left(\boldsymbol{n}_{i}-1\right)\right] .
$$

The first term describes the coupling between neighbouring sites with a strength $J$. We use cyclic boundary conditions, i.e. hopping between the first and the last site of the lattice is included. The last two terms of (2) give the on-site singleparticle energy $\epsilon_{i}$ and the on-site two-body interaction with a strength $V$. Formally, the parameters are given by matrix elements of components of the coordinate space Hamiltonian calculated with the Wannier wavefunctions associated with the individual sites [8]. The hopping strength $J$, for example, is the off-diagonal matrix element of the kinetic energy operator calculated in the Wannier basis. For an irregular lattice potential the on-site energies $\epsilon_{i}$ depend explicitly on the site index $i$; for simplicity we neglect the site-dependence of $J$ and $V$. Interactions between particles at different sites and long-range hopping are also neglected.

To determine the ground state of the system we solve the eigenvalue problem of the Bose-Hubbard Hamiltonian numerically. The Hamilton matrix in the number basis is easy to calculate; the on-site energy and the two-body interaction form the diagonal and the hopping term generates a few off-diagonal matrix elements. Since the Hamilton matrix is very sparse and we are interested in the lowest eigenstates only, an iterative Lanczos-type algorithm is most efficient to solve the eigenvalue problem. This enables us to treat systems with up to $I=12$ and $N=12$ on a standard PC without further approximations. Larger systems can be treated by Monte Carlo techniques [9, 10]. Two relevant ground state observables we consider in the following are the mean occupation number $\bar{n}_{i}=\left\langle\psi\left|\boldsymbol{n}_{i}\right| \psi\right\rangle$ and number fluctuations $\sigma_{i}^{2}=\left\langle\psi\left|\boldsymbol{n}_{i}^{2}\right| \psi\right\rangle-\left\langle\psi\left|\boldsymbol{n}_{i}\right| \psi\right\rangle^{2}$ at the individual sites.

\section{Mott insulator transition}

The interplay between the three terms of the Bose-Hubbard Hamiltonian (2) generates a rich zero-temperature phase diagram. Its basic structure can be understood by analysing the contributions of the different terms to the energy expectation value for particular states. The off-diagonal hopping term gives a negative contribution to the total energy if the state is a superposition of many number states. If only the hopping is present the coefficients of the ground state (1) are related to the multinomial coefficients, thus all number states contribute and number fluctuations $\sigma_{i}$ are large. The particles can tunnel freely through the lattice and the system resembles a superfluid (SF).

If a repulsive two-body interaction is included then number states with large occupation numbers at individual sites have high energy expectation values. The repulsion favours homogeneous distributions of the particles over all sites. The competition between hopping term and two-body interaction governs one kind of quantum phase transition present in these systems. For small interaction strengths $V / J$ the hopping term dominates and the ground state is a superposition of

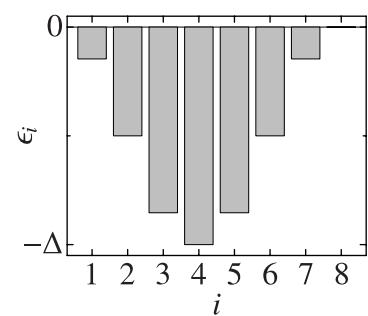

Figure 1. On-site energies $\epsilon_{i}$ for a unit cell of the sinusoidal superlattice.

many number states - a superfluid. With increasing $V / J$ those number states with large occupation numbers at some sites are gradually suppressed because of their large interaction energy.

For integer (commensurate) fillings $N / I$ there is a unique number state with $n_{i}=N / I$ at each site which minimizes the expectation value of the two-body interaction. For sufficiently strong interactions the ground state is given by just this number state. Therefore number fluctuations $\sigma_{i}$ and also the expectation value of the hopping term vanish. This phase in which tunnelling of the particles is inhibited by the repulsive interaction is called the Mott insulator (MI) phase. Quantum Monte Carlo calculations for the infinite one-dimensional Bose-Hubbard model with $N / I=1$ show that the SF to MI transition occurs at $(V / J)_{\text {crit }} \approx 4.65[10]$, which is confirmed by a renormalization group study [11] and a strong-coupling expansion [4]. Mean-field models predict a much larger critical interaction strength $[8,12]$.

\section{Two-colour superlattices}

How does disorder affect the phase diagram of the BoseHubbard model? Most of the theoretical investigations on disorder-induced effects concentrate on infinite lattices with completely random on-site energies. More relevant for cold bosonic atoms in optical lattices are superlattice structures, i.e. lattices composed of irregular unit cells. As the simplest example we discuss a one-dimensional optical superlattice with a sinusoidal modulation of the on-site energies $\epsilon_{i}$. A unit cell consists of $I=8$ lattice sites and the $\epsilon_{i}$ vary in the interval $[-\Delta, 0]$ as shown in figure 1 . Experimentally this modulation can be realized using a superposition of two optical standing waves with appropriate wavelengths - a so-called two-colour lattice. This allows the independent control of the tunnelling strength $J$ and the modulation amplitude $\Delta$. The sinusoidal modulation already shows the relevant fundamental features but is only the most simple realization of disorder ${ }^{1}$, more complex superlattices have already been generated experimentally [7].

Let us start from a noninteracting $\mathrm{SF}$ in a regular lattice $(\Delta / J=0, V / J=0)$ and increase the modulation amplitude $\Delta$ gradually. The mean occupation number $\bar{n}_{i}$ of the site with lowest $\epsilon_{i}$ in each unit cell will increase. For sufficiently strong disorder the ground state will be composed of number states which have non-zero occupation only at the deepest well of each unit cell. This mechanism is similar to Anderson localization in an infinite system with completely random

1 We use the term disorder not just for completely random lattice potentials but in a broader sense which comprises all kinds of non-uniform lattices. 

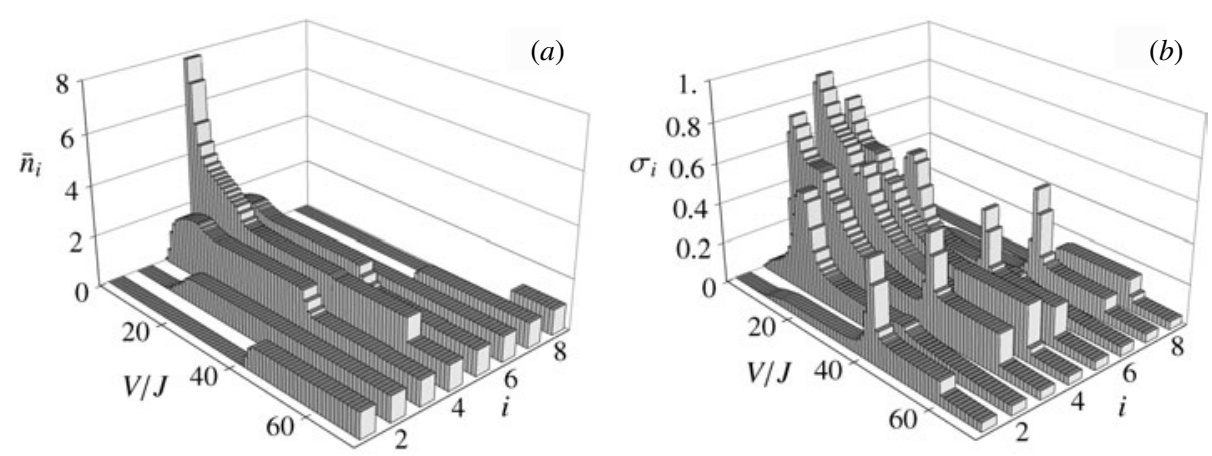

Figure 2. Mean occupation numbers $\bar{n}_{i}(a)$ and number fluctuations $\sigma_{i}(b)$ for the sinusoidal superlattice with $I=8, N=8$, and $\Delta / J=60$ as function of $V / J$. The different rows correspond to the individual sites of a unit cell.

on-site energies [13]. However, in contrast to the Anderson localized phase in a random lattice the ground state is still a superposition of several number states with considerable number fluctuations $\sigma_{i}$. Therefore, this state appears almost like a SF state in a regular lattice with $I$ times the lattice spacing.

The disorder-induced localization is strongly affected by repulsive interactions which drive the system towards even distributions of particles over all sites. To study the detailed interplay between interactions and disorder we diagonalize the Hamiltonian numerically for an isolated $I=8$ unit cell. This assumes that the exchange of particles between different unit cells can be neglected. By direct comparison with two-cell calculations this turns out to be a very good approximation for $V / J>0$. For the noninteracting system, however, the results of single- and multi-cell calculations differ. The ground state obtained in a multi-cell calculation exhibits the large fluctuations discussed previously. A diagonalization using a single unit cell will lead, in the limit of large $\Delta$, to a localized state with vanishing fluctuations.

Figure 2 shows the mean occupation numbers $\bar{n}_{i}$ and the number fluctuations $\sigma_{i}$ for the sinusoidal superlattice with $\Delta / J=60$ and commensurate filling $(I=8, N=8)$ as function of the interaction strength $V / J$. For vanishing interaction strength all particles are localized at the lowest energy site within the unit cell. However, with increasing $V / J$ the mean occupation at this site is reduced rapidly and the particles are redistributed to sites with higher on-site energies. This rearrangement happens stepwise, i.e. first the two sites with second lowest $\epsilon_{i}$ are populated. Then at $V / J \approx 10$ the population of the next two sites increases, and so on. Between successive rearrangements there are extended plateaux (e.g. for $20<V / J<40$ ) with constant and approximately integer mean occupation numbers $\bar{n}_{i}$. This characteristic sequence of rearrangements and stable plateaux is also reflected in the number fluctuations $\sigma_{i}$ shown in figure $2(b)$. Within the rearrangement regions number fluctuations of those sites which change their mean occupation number are large. Within the plateaux all $\sigma_{i}$ are small and the ground state is almost a pure number state. This region of successive rearrangements is called the Bose glass (BG) phase [2, 3]. In this phase the energy gap, that is, the energy difference between the ground state and the first excited state, is small unlike in the MI phase. At the same time the SF density vanishes, i.e. the system is an insulator. We will discuss these observables in more detail in a forthcoming publication.
Eventually, at $V \approx \Delta$ a final rearrangement occurs and all sites have equal mean occupation and very small number fluctuations. This is the transition from the BG to the MI phase where the ground state is a pure number state with $n_{i}=1$ at each site.

Figure 3(a) summarizes the phase diagram for the sinusoidal superlattice. The contour plot shows the square of the largest coefficient $C_{\max }^{2}=\max \left(C_{\alpha}^{2}\right)$ in the expansion (1). The dark shadings indicate that the ground state is a pure number state. The MI phase appears only for $V>\Delta$. For interaction strengths below this boundary two stable configurations within the BG phase follow. At very small $V / J$ and large disorder amplitudes the localized phase (L) appears. The remaining regions resemble a (disordered) SF. A complementary representation of the phase diagram is shown in figure $3(b)$ in terms of the maximum on-site number fluctuations $\sigma_{\max }=\max \left(\sigma_{i}\right)$. Large number fluctuations indicate that the ground state is a superposition of many number states, i.e. that $C_{\max }^{2}$ is small.

The structure of the BG phase, i.e. the sequence of rearrangements, depends strongly on the particular pattern of on-site energies. More complex modulations of the $\epsilon_{i}$ generate a larger variety of configurations in the BG phase. The same holds true for non-commensurate fillings. In the sinusoidal lattice with $N=9$, extended regions emerge, where two sites exhibit mean occupation $\bar{n}_{i}=0.5$ or 1.5 in association with large number fluctuations.

\section{Matter-wave interference pattern}

How can these structures be observed experimentally? An experimentally quite simple approach is the imaging of matter-wave interference patterns after release from the trap and ballistic expansion [6, 14]. The crucial quantity that determines the presence or absence of an interference pattern is the phase coherence between atoms at different lattice sites. Small phase fluctuations at the individual sites, which imply phase coherence, are connected to large number fluctuations $\sigma_{i}$. An estimate for the phase fluctuations $\sigma_{\phi, i}$ can be obtained from the relation $\sigma_{\phi, i}=1 /\left(2 \sigma_{i}\right)$ [14]. To simulate the density interference pattern for a given ground state we approximate the wavefunction of an atom localized at a lattice site by a Gaussian wavepacket ${ }^{2}$. The superposition of the

2 The centroids of the Gaussians for the different sites have a constant distance $\delta \xi$. The width $\sqrt{0.025} \delta \xi$ is chosen such that the typical three-peak structure for the SF phase [6] is reproduced. 
(a)

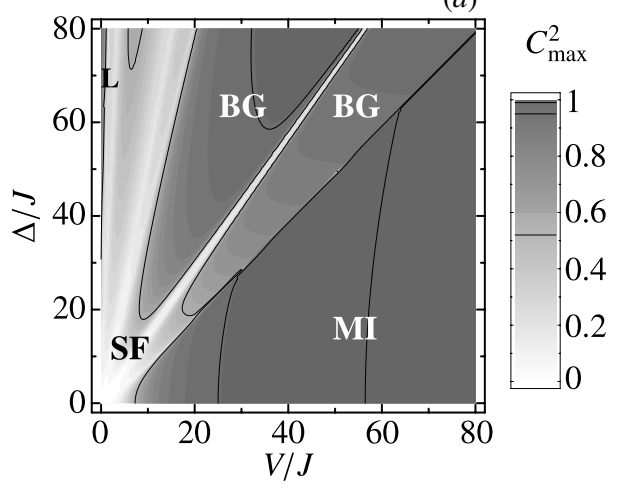

(b)

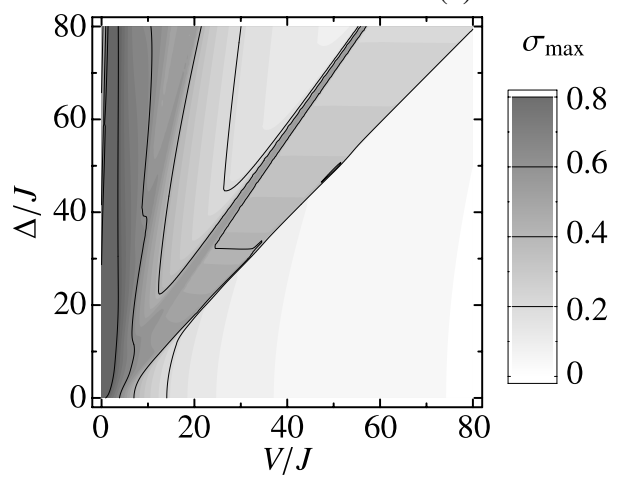

Figure 3. (a) Contour plot of the square of the largest coefficient $C_{\max }^{2}$ for the sinusoidal superlattice with $I=8, N=8$ as function of $V / J$ and $\Delta / J$. Dark shadings indicate that the ground state is a pure number state (insulators). The labels identify the different phases: superfluid $(\mathrm{SF})$, Mott insulator (MI), Bose glass (BG), and localized (L). (b) Analogous plot for the maximum on-site number fluctuations $\sigma_{\text {max }}$.

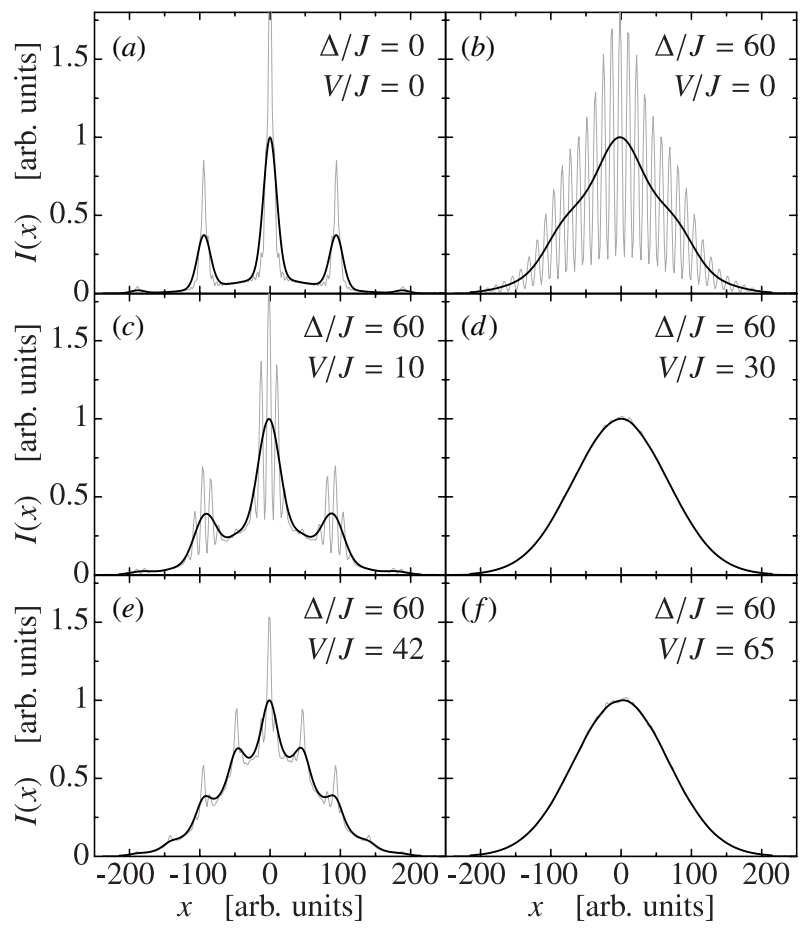

Figure 4. Matter-wave interference patterns for different combinations of $V / J$ and $\Delta / J$. The grey curves show the full interference pattern of the superlattice; the black curves result from folding with a Gaussian profile to mimic a restricted experimental resolution.

wavepackets after free expansion (neglecting interactions) results in a density interference pattern. To account for the phase fluctuations we calculate the incoherent average over typically 10000 sets of on-site phases, which are chosen randomly with a Gaussian distribution of width $\sigma_{\phi, i}$.

Figure 4 depicts characteristic interference patterns for different points of the phase diagram. The superlattice character is explicitly included by replicating the unit cell. This gives rise to the short-wavelength oscillations which reflect the periodicity of the superlattice. The black curves are obtained by folding with a Gaussian profile to mimic a restricted experimental resolution. The remaining smooth pattern is largely determined by the structure within the unit cells.
In the absence of interactions and disorder we obtain the prominent interference pattern of the SF [6] with pronounced peaks shown in figure $4(a)$. This structure dissolves if we enter the localized phase shown in panel $(b)$ for $\Delta / J=60$; depending on the experimental resolution one may detect the interference pattern of a lattice with $I$ times the fundamental lattice spacing. If we include interactions $V / J>0$ and enter the BG phase we observe a characteristic vanishing and reappearance of interference fringes which is correlated to the number fluctuations shown in figure $2(b)$. For weak interactions $V / J=10$ the three-peak structure of the SF reappears with broader peaks and increased incoherent background due to the reduced number fluctuations. In the stable regions of the $\mathrm{BG}$ phase, e.g. at $V / J=30$, number fluctuations of all sites are small and the fringes vanish again; only the incoherent background remains, as shown in panel $(d)$. In the vicinity of rearrangements certain lattice sites regain large number fluctuations and cause the reappearance of interference fringes. Figure 4(e) depicts an example for $V / J=42$, where every second lattice site has considerable fluctuations (compare figure 2) which create a distinctive fringe pattern. Eventually, if we enter the MI phase only the incoherent bump remains, as shown in panel $(f)$. To obtain complementary information on the spatial density distribution of these disordered states Bragg diffraction $[7,15,16]$ would be a useful tool.

\section{Summary}

In summary, we have shown that ultracold atomic gases in optical superlattices are an ideal system to study the complicated interplay between interaction and disorder. As a function of the interaction strength one can observe the detailed evolution of the ground state from an spatially localized state through a BG to the MI, which is accompanied by a characteristic vanishing and reappearance of interference fringes. The remarkable degree of experimental access to all relevant parameters allows a comprehensive study of these quantum phase transitions.

\section{Acknowledgments}

This work was supported by the Deutsche Forschungsgemeinschaft, the UK EPSRC, and the EU through the Cold Quantum Gases Network. 


\section{References}

[1] Fisher M P A, Weichman P B, Grinstein G and Fisher D S 1989 Boson localization and the superfluid-insulator transition Phys. Rev. B 40546

[2] Scalettar R T, Batrouni G G and Zimanyi G T 1991 Localization in interacting, disordered, Bose systems Phys. Rev. Lett. 663144

[3] Krauth W, Trivedi N and Ceperley D 1991

Superfluid-insulator transition in disordered boson systems Phys. Rev. Lett. 672307

[4] Freericks J K and Monien H 1996 Strong-coupling expansion for the pure and disordered Bose-Hubbard model Phys. Rev. B 532691

[5] Rapsch S, Schollwöck U and Zwerger W 1999 Density matrix renormalization group for disordered bosons in one dimension Europhys. Lett. 46559

[6] Greiner Mo et al 2002 Quantum phase transition from a superfluid to a Mott insulator in a gas of ultracold atoms Nature $\mathbf{4 1 5} 39$

[7] Guidoni L, Triché C, Verkerk P and Grynberg G 1997 Quasiperiodic optical lattices Phys. Rev. Lett. 793363
[8] Jaksch D et al 1998 Cold bosonic atoms in optical lattices Phys. Rev. Lett. 813108

[9] Batrouni G G et al 2002 Mott domains of bosons confined on optical lattices Preprint cond-mat/0203082

[10] Batrouni G G and Scalettar R T 1992 World-line quantum Monte Carlo algorithm for a one-dimensional Bose model Phys. Rev. B 469051

[11] Singh K G and Rokhsar D S 1992 Real-space renormalization study of disordered interacting bosons Phys. Rev. B 463002

[12] Krauth W, Caffarel M and Bouchaud J-P 1992 Gutzwiller wavefunction for a model of strongly interacting bosons Phys. Rev. B 453137

[13] Belitz D and Kirkpatrick T R 1994 The Anderson-Mott transition Rev. Mod. Phys. 66261

[14] Orzel C et al 2001 Squeezed states in a Bose-Einstein condensate Science 2912386

[15] Weidemüller M et al 1995 Bragg diffraction in an atomic lattice bound by light Phys. Rev. Lett. 754583

[16] Birkl G et al 1995 Bragg scattering from atoms in optical lattices Phys. Rev. Lett. 752823 